\title{
A new Arabic stemming algorithm
}

Eiman Tamah AlShammari and Jessica Lin

Department of Computer Science, George Mason University, USA

https://doi.org/10.36505/ExLing-2008/02/0004/000063

\begin{abstract}
Text processing is a vital step in the information retrieval process, text mining, and natural language processing. It includes several stages, such as normalization, stop word removal, and stemming. Stemming is the process of reducing the lexicon to its root. Due to the different structures and rules in languages, the task of stemming is language-dependent. This research introduces a new stemming algorithm for the Arabic Language. Arabic is one of the most complex languages, both spoken and written. However, it is also one of the most common languages in the world. It is the base from which many other languages are derived. Despite the wide usage of the language, technology and development for Arabic has been limited. The main reason lies within the formulation rules of Arabic, as Arabic language exhibits a very complicated morphological structure. Existing Arabic stemmers suffer from high stemming error-rates. They blindly stem all the words and perform poorly, especially with compound words, proper nouns and foreign Arabized words. The main cause of this problem is the stemmer's lack of knowledge of the word lexical category (i.e. noun, verb, proposition, etc.) This paper presents a new stemming algorithm that relies on Arabic language morphology and Arabic language syntax. The automated addition to the syntactic knowledge reduced both stemming error and stemming cost. Additionally, the new Algorithm automatically creates it is own list of proper nouns, and compound words based on the processed corpus.
\end{abstract}

Key words: Arabic, stemming, morphology

\section{Introduction}

The efforts to improve Arabic information search and retrieval compared to other languages are limited and modest, even though the Arabic language is the official language for over 29 countries, in addition to which there are native Arabic speakers scattered all over the world. The barrier to text processing advancements in Arabic is its very complicated morphological structure.

Stemming Arabic documents was done manually prior to TREC (Text Retrieval Conference). The two most effective Arabic stemmers are Larkey's light stemmer (Larkey and Connell 2001; Larkey, Ballesteros and Connell 2002) and Khoja's (Khoja 1999) root-extraction stemmer.

Over-stemming and under-stemming are the main drawbacks of the rootbased stemming and the light stemming algorithms, respectively. Overstemming, under-stemming and mis-stemming are all stemming errors that usually degrade the correctness of stemming algorithms (Baeza-Yates 1992).

ExLing 2008: Proceedings of 2nd Tutorial and Research Workshop on Experimental Linguistics, 25-27 August 2008, Athens, Greece 
To decrease stemming errors, other stemmers add a lookup dictionary to check the roots after stemming. This process is computationally expensive; Al-Fedaghi and Al-Anzi (Al-Fedaghi and Al-Anzi 1989) estimated that there are around 10,000 independent roots and each root word can have prefixes, suffixes, infixes, and regular and irregular tenses.

To mitigate the drawbacks of the previous work on Arabic stemming, we propose an alternative that defines a rule to stem words instead of chopping off the letters. This rule is set by the syntactical structure of the word. For example verbs require aggressive stemming and need to be represented by their roots. Nouns on the contrary only require light suffixes and prefixes elimination.

\section{Arabic language structure}

Arabic language is a semantic language with a composite morphology. The words are categorized as particles, nouns, or verbs. There are 28 letters in Arabic, and the words are formed by linking letters of the alphabet. Letters of the alphabet differ in shape based on their position within the word (i.e. beginning, middle, or end). Unlike most Western languages, Arabic script is written from right to left. Furthermore, proper nouns do not start with capital letters, thus, extracting nouns and proper nouns is a challenging task for machines.

Also, in English, words are formed by attaching prefixes and suffixes to either or both sides of the root. For example the word Untouchables is formed as follows:

\begin{tabular}{|l|l|l|l|}
\hline Un & touch & able & s \\
\hline Prefix & Root & First Suffix & Second Suffix \\
\hline
\end{tabular}

In Arabic, additions to the root can be within the root (not only on the word sides) which is called a pattern. This causes a serious issue in stemming Arabic documents because it is hard to differentiate between root particles and affix letters. For example, for the root "drink" شرب in Arabic, adding the letter “" (infix) formed a different words such as شارب "drinker" can be formed by adding the letter "”" (infixes).

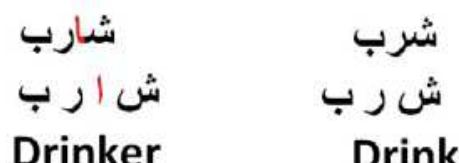

Figure 1. Arabic infix example. 
Suffixes, prefixes and infixes are categorized based on their use. Similar to other Western languages, there are specific suffixes to convert the word from the singular form to the plural form and others to convert from masculine to feminine.

\section{Arabic stemming algorithm}

Table 1. Arabic Stemming Algorithm.

Input: Arabic document

Output: Stemmed document, Noun Dictionary, Verbs Dictionary

V: Verb dictionary (one dimensional array sorted alphabetically ${ }^{1}$ )

$\mathrm{N}$ : Noun dictionary (one dimensional array sorted alphabetically)

NSW: Array of stop words proceeding nouns

VSW: Array of stop words proceeding verbs

SW: Array of stop words (including both NSW and VSW)

Phase Zero: Remove useless stop words.

Phase One: Simple Noun Identification and Noun Dictionary Generation

Locate words attached to definite articles, and preceded by NSW and flag them as Nouns, Add the identified words to N.

Phase Two: Suffix and Prefix removal

Apply suffix and prefix approach to the entire document. Longest suffixes and prefixes are removed first.

Phase Three: Verbs Identification and Verb Dictionary Generation

Verbs proceeded by VSW are flagged and added to the V

Phase Four: Find all noun tokens

Phase Five: Stop Word Removal : Remove useful and useless stop words

Phase Six: Root Extraction for Verbs

Phase Seven: Roots are extracted by comparing Verbs to Arabic Root patterns; words with missing tags are considered nouns and lightly stemmed.

Our novel algorithm consists of different phases. During the first phase, useless stop words are removed to reduce the size of the corpus. Next, we identify nouns by either locating Stop words that always precede nouns (example: the, and a) or words starting by definite articles. These nouns are lightly stemmed by removing suffixes and prefixes and then added to a global nouns dictionary. At this level, these words are flagged as nouns as a preparation to the stemming phase. In parallel to that process we find verbs by locating stop words that always precede verbs. Similar to the nouns, the 
verbs are added to a global verb dictionary and tagged as verbs. In Arabic, we cannot have two consecutive verbs, thus any word following a verb is either a stop word or a noun. If the word is not a stop word then the word is added to the noun dictionary and flagged as a noun.

Before we direct a word to the appropriate stemming by the word flag, all the stop words are removed since they offer no further advantage. The document is revisited by categorizing words with missing flags using the noun corpus and the verb corpus as a lookup table. Other words that do not belong in any category will be treated as nouns and stemmed lightly. Table 1 below summarizes the algorithm.

\section{Notes}

${ }^{1}$ For fast lookup, these dictionaries can be implemented using hash tables.

\section{References}

Al-Fedaghi, S.S. and Al-Anzi, F., 1989. A New Algorithm to Generate Arabic RootPattern Forms. Proceedings of the 11th National Computer Conference and Exhibition, 391-400.

Baeza-Yates, R., 1992. Text retrieval: Theory and practice. 12th IFIP World Computer Congress, 1, 465-476.

Khoja, S., 1999. Stemming Arabic Text. Lancaster, UK, Computing Department, Lancaster University,

Larkey, L.S., Ballesteros, L. and Connell, M.E., 2002. Improving stemming for Arabic information retrieval: light stemming and co-occurrence analysis. Proceedings of the 25th annual international ACM SIGIR conference on Research and development in information retrieval, 275-282.

Larkey, L.S. and Connell, M.E., 2001. Arabic Information Retrieval at UMass in TREC-10. Proceedings of the Tenth Text REtrieval Conference (TREC-10)", EM Voorhees and DK Harman ed, 562-570. 